\title{
Japanese VLBI network observations of 6.7-GHz methanol masers II. Results
}

\author{
Koichiro Sugiyama ${ }^{1}$, Kenta Fujisawa ${ }^{1}$, Mareki Honma ${ }^{2}$, Akihiro Doi ${ }^{3}$, \\ Nanako Mochizuki ${ }^{3}$, Yasuhiro Murata ${ }^{3}$ and Yasuko Isono ${ }^{1}$ \\ ${ }^{1}$ Faculty of Science, Yamaguchi University, \\ 1677-1 Yoshida, Yamaguchi, Yamaguchi 753-8512 \\ ${ }^{2}$ National Astronomical Observatory of Japan, 2-21-1 Osawa, Mitaka, Tokyo 181-8588 \\ ${ }^{3}$ The Institute of Space and Astronautical Science, Japan Aerospace Exploration Agency, \\ 3-1-1 Yoshinodai, Sagamihara, Kanagawa 229-8510
}

\begin{abstract}
We are using a Japanese VLBI network (JVN) for VLBI observations of 6.7-GHz methanol masers associated with massive star-forming regions. Here we present results for Cepheus A (Cep A) from observations taken on September 9, 2006. The distribution of the maser spots indicates either a spherical bubble or a disk having an inclination of several tens degree. We construct a disk model with an inclination of $\sim 70^{\circ}$ and a radius of $\sim 700 \mathrm{AU}$. From a luminosity of $1.7 \times 10^{4} L_{\odot}$ for a source observed in this region, the excitation of the maser is probably radiative.
\end{abstract}

Keywords. masers, stars: formation, HiI regions, ISM: individual (Cepheus A)

\section{Introduction}

Methanol masers at $6.7 \mathrm{GHz}$ provide a useful probe for investigating star formation associated with massive young stellar objects (YSOs). The masers often show linear structures and linear velocity gradients, and these are often interpreted as evidence for edge-on disks (e.g., Pestalozzi et al. 2004). However, other morphologies could also explain the maser distributions.

There are 519 known sites of $6.7 \mathrm{GHz}$ methanol maser listed (Pestalozzi et al. 2005), while the number of sources observed with VLBI at milli-arcsecond (mas) resolution is, currently, less than 30. We have begun VLBI observations of maser sources at $6.7 \mathrm{GHz}$ with a Japanese VLBI network (JVN; Doi et al. 2006) with five antennas, Yamaguchi $32 \mathrm{~m}$, Usuda $64 \mathrm{~m}$, VERA-Mizusawa, Ishigaki and Iriki $20 \mathrm{~m}$.

In this paper, we describe results from observations of Cepheus A (Cep A). The observations were taken on September 9, 2006. Cep A has a CO condensation at a distance of $730 \mathrm{pc}$ (Johnson 1957) and is known to have some massive star forming regions. One of these is an UC Hit region, Cep A-HW 2, which is the site of the methanol maser emission. It is also known that a biconical ionized jet exists in Cep A-HW 2 from radio continuum observations (Torrelles et al. 1996).

\section{Results and discussion}

The distribution of maser spots of Cep A (Figure 1) shows an incomplete arc which could be part of a spherical bubble, or a disk with an inclination of several tens degree. We assume here that the distribution of spots traces a tilted disk because of the nearly perpendicular direction to the radio jet. We also assume that the maser spots are excited in regions of the same radiation temperature, i.e. that they are at the same distance from 

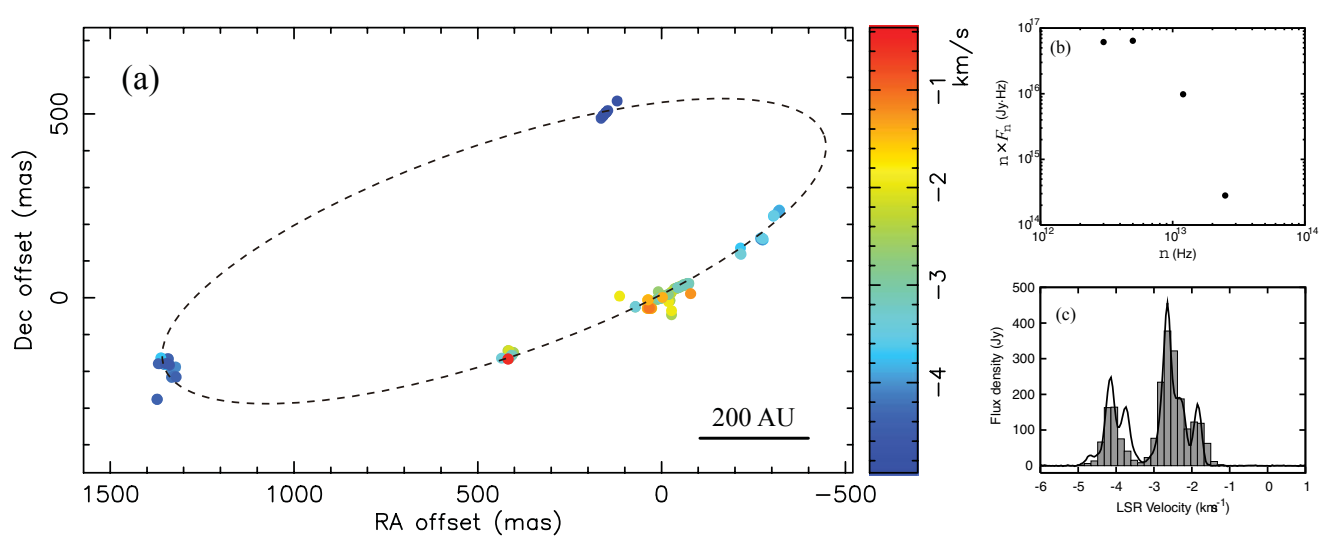

Figure 1. Cep A: (a) distribution of methanol maser at $6.7 \mathrm{GHz}$. (b) IRAS flux at each wavelength $(12,25,60,100 \mu \mathrm{m})$ versus frequency for IRAS $22543+6145$. (c) The filled box shows the CLEANed component spectrum while the solid line shows the single-dish (total-power) spectrum.

an excitation source. According to this model, we have fitted the distribution with an ellipse and obtained a radius of $R \sim 700 \mathrm{AU}$ with an inclination of $\sim 70^{\circ}$. The radiation temperature at this radius is estimated as follows. We first calculate the luminosity of the (suspected) excitation source of an IRAS point source (IRAS 22543+6145) located at Cep A-HW 2, by using formula (3) from Walsh et al. (1997). For a distance to Cep A of $730 \mathrm{pc}$, a luminosity of $L=1.7 \times 10^{4} L_{\odot}$ is obtained. We can then derive the radiation temperature $T_{\mathrm{d}}$ with:

$$
T_{\mathrm{d}}=(16 \pi \sigma)^{-1 / 4} \cdot R^{-1 / 2} \cdot L^{1 / 4}
$$

where $\sigma$ is the Stefan-Boltzmann constant. The temperature obtained, $T_{\mathrm{d}} \sim 120 \mathrm{~K}$, agrees well with a theoretical value $>100 \mathrm{~K}$ (Cragg et al. 2002). This is consistent with a single excitation source and the methanol maser spots in Cep A distributed within a disk of diameter $1400 \mathrm{AU}$.

\section{Acknowledgements}

The JVN is led by the National Astronomical Observatory of Japan (NAOJ), Hokkaido University, Gifu University, Yamaguchi University, and Kagoshima University, in cooperation with Geographical Survey Institute (GSI), the Japan Aerospace Exploration Agency (JAXA), and the National Institute of Information and Communications Technology (NICT).

\section{References}

Cragg, D. M., Sobolev, A. M., \& Godfrey, P. D. 2002, MNRAS, 331, 521

Doi, A., et al. 2006, Proc. 8th European VLBI Network Symposium, PoS(8thEVN) 071

Johnson, H. L. 1957, ApJ, 126, 121

Pestalozzi, M. R., Elitzur, M., Conway, J. E., \& Booth, R. S. 2004, ApJ, 603, L113

Pestalozzi, M. R., Minier, V., \& Booth, R. S. 2005, A\&A, 432, 737

Torrelles, J. M., Gomez, J. F., Rodriguez, L. F., Curiel, S., Ho, P. T. P., \& Garay, G. 1996, ApJ, 457, L107

Walsh, A. J., Hyland, A. R., Robinson, G., \& Burton, M. G. 1997, MNRAS, 291, 261 